Classification

Physics Abstracts

$61.16 \mathrm{D}$

\title{
High-resolution electron microscopy of fine particles
}

\author{
M. Mitome $\left({ }^{2}\right)$, Y. Ohshima $\left({ }^{3}\right)$, M. Itoh $\left({ }^{1}\right)$ and K. Takayanagi $\left({ }^{1}\right)$ \\ ( $\left.{ }^{1}\right)$ Tokyo Institute of Technology, Materials Science and Engineering, 4259 Nagatsuda, Midori-ku, \\ Yokohama, 227, Japan \\ ( ${ }^{3}$ CANON Research Center, Morinosato-Wakamiya, Atsugi-Shi, Kanagawa, 243-01, Japan \\ $\left({ }^{3}\right)$ Fujitsu Laboratories Ltd. 10-1 Morinosato-Wakamiya, Atsugi, 243-01, Japan
}

(Received March 17, 1993; accepted April 22, 1993)

\begin{abstract}
Structures of fine particles which contains $30-10^{4}$ atoms have been investigated by UHV high-resolution electron microscopy. Three topics on structural anomaly of fine particles are described and discussed: structural instability, surface anomaly, and a new intermediate phase related with surface melting. Anomalous surface reconstruction is generated on a (111) facet of Ge particle, which has not been found on the bulk surface of the same orientation, as well as fine particles of $\mathrm{Au}$ have specific $n \times 1(n=3,5,7)$ surface reconstructions on (100) facet. In the case of Sn particle, no apparent surface reconstructions have been recognized, while some irregularity of the surface layer have been noticed for particles less than the critical size $(2 r=5 \mathrm{~nm})$, which indicates surface disordering or melting. Below the critical size appears an intermediate phase between liquid and solid phase, where crystal embrios seem to be generated and annihilated within the particle. To determine structures and dynamic changes of fine particles with such irregularity, a new imaging method of highresolution electron microscopy has been proposed: A convergent beam illumination (CBI), instead of parallel beam illumination (PBI), produces atom image at the right position even specimens of an irregular structure.
\end{abstract}

\section{Introduction.}

Fine particles attract much interest because of their specific properties different from the bulk materials. The specific properties arise due to surface effect and/or finite number effect of fine particles. Effects due to finite number of electrons in fine particles have been discussed first by Kubo [1] to find anomalies in susceptivities. Less understood are surface effects, because they depend on the surface structure of each fine particle which is difficult to be analyzed.

Electron microscopy is one of the direct method for structure analysis of fine particles. Fine particles of sizes $1 \mathrm{~nm}-10 \mathrm{~nm}$ contains $30-10^{4}$ atoms in them. Therefore, roughly $70 \%-10 \%$ of total atoms belong in the surface, so that the surface should play an important role in determining their specific electronic and optical properties [2]. To analyze atomic positions through electron microscope images, one needs computer simulation of the structure [3]. It is not straightforward to assign a simulated image to an observed image of a fine particle, because of some irregular structure [4]. Therefore, we need a new imaging method which enables us to determine the structure 
directly from a microscope image directly and uniquely.

In the following sections, we describe first a new imaging method $[4,5]$, and then summarize our recent findings on the imaging method and some structural properties of fine particles [6]: surface anomaly of Ge particle [7], irregular surface structure of Sn particles of a critical size, and an intermediate phase between the liquid and solid phase [8].

\section{Convergent beam illumination.}

High-resolution imaging is used to be done by a parallel beam illumination where the incident beam illuminates the specimen as parallel as possible with a beam divergence less than $1 \mathrm{mrad}$. Here we summarize the new imaging method using convergent beam illumination (CBI) reported in previous papers. Mitome $e t$ al. have proposed a convergent beam illumination to improve the resolution. The convergent beam illumination not only improves the resolution by $0.3 \mathrm{~A}$ from that of the PBI illumination, but also can image each atom at the right position even for specimens with non-periodic structures. An optimum condition for the convergent beam illumination has been found to be at the beam divergence of $q_{\mathrm{s}}^{*}=q(\operatorname{Cs} \lambda)^{1 / 4}=0.4$, and defocus of $\Delta_{f}^{*}=\Delta f /(\operatorname{Cs} \lambda)^{1 / 2}=$ 1.4. Theoretical consideration results in the following simple expression of an atom image profile for the CBI imaging: Provided that $j-$ th atom of an atomic potential $V(r)$ is located at $r_{j}$ in a fine particle, the CBI image is such that the $j$-th atom image appear with the image profile given by

$$
U(r)=U_{\text {thin }}(r)+\tan (\varphi) U_{\text {thick }}(r)
$$

where $\varphi$ is the phase angle of the transmitted wave,

$$
U_{\text {thin }}(r)=2 \pi \sigma \int B_{1}(k) f(k) J o(2 \pi k r) k \mathrm{~d} k
$$

and

$$
U_{\text {thick }}(r)=2 \pi \sigma \int B_{2}(k) f(k) J o(2 \pi k r) k \mathrm{~d} k
$$

where $f(k)$ is an atom form factor of the $j$ - th atom, and

$$
\begin{aligned}
& B_{1}(k)=2 \int s(q) \sin \{\chi(q+k)-\chi(q)\} \mathrm{d} q \\
& B_{2}(k)=2 \int s(q) \cos \{\chi(q+k)-\chi(q)\} \mathrm{d} q
\end{aligned}
$$

In equations ( $3 a$ ) and ( $3 b$ ) beam divergence of the partially coherent incident beam is defined by a Gaussian function as

$$
s(q)=\frac{1}{\pi q_{s}^{2}} \exp \left\{-\frac{q^{2}}{q_{s}^{2}}\right\}
$$

and the phase shift of electron waves by the objective lens with a spherical aberration $C_{\mathrm{s}}$, is given by

$$
\chi(k)=\frac{1}{4} C_{\mathrm{s}} \lambda^{3} k^{4}-\frac{1}{2} \Delta f \lambda k^{2}
$$

As easily understood from equation (1), atom image profile varies depending on the thickness of the specimen. $U_{\text {thin }}(r)$ and $U_{\text {thick }}(r)$ are independent of the thickness, but are determined only by the imaging condition of the electron microscope (beam divergence, defocus,...) and the atom 
species through $f(k)$. Thickness dependence of the atom image comes from the term, $\tan (\varphi)$. The optimum condition of the CBI imaging has been, then, determined such that the atom image profile $U(r)$ is less sensitive to the thickness change, and has a simple form like Gaussian function. In case of PBI imaging, $U_{\text {thin }}(r)$ has a Gaussian form with oscillatory tail, while $U_{\text {thick }}(r)$ oscillates and deteriorates the image profile of the atom. On the other hand, in case of CBI imaging, $U_{\text {thin }}(r)$ and $U_{\text {thick }}(r)$ vary less oscillatory than PBI imaging. As the thickness increases $U_{\text {thick }}(r)$ contributes more to $U(r)$ and obscures Gaussian form of the atom image. Maximum thickness that $U(r)$ keeps Gaussian form is estimated to be $\tan (\varphi) \lesssim 1$ [5]: The maximum thickness is about $5 \mathrm{~nm}$ for Au, $7 \mathrm{~nm}$ for $\mathrm{Pb}[5]$. As is seen in figure 1, atom image profile for the PBI imaging with a beam divergence of $q_{\mathrm{s}}^{*}=0.1$ is much oscillatory compared with that for CBI imaging, so that subsidery peaks in $U(r)$ can give rise to some pseudo- atom image at positions of nearest neighbour atoms.

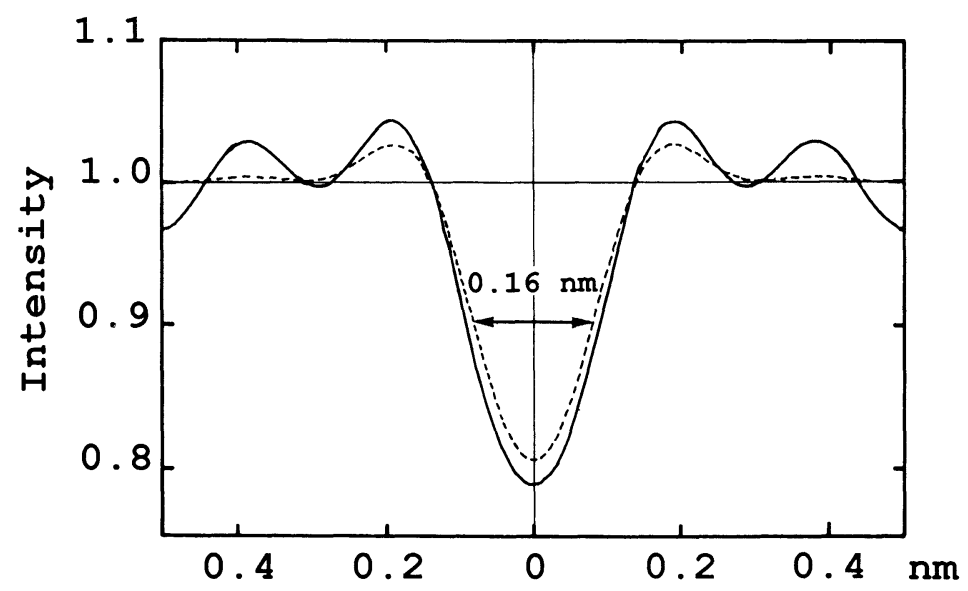

Fig. 1. - Atom image profile calculated under PBI condition $\left(q_{\mathrm{s}}^{*}=0.1:\right.$ solid line $)$ and CBI condition $\left(q_{\mathrm{s}}^{*}=0.1:\right.$ dotted line). Note that the image profile of CBI (half width is $0.16 \mathrm{~nm}$ ) is sharper than that of PBI (half width is $0.18 \mathrm{~nm}$ ), which indicates improvement of the resolution of the electron microscope by using $\mathrm{CBI}$ imaging.

The CBI imaging has an advantage that one can obtain reasonable resolution at lower acceleration voltage of the microscope, which reduces damages of specimens due to high-energy electrons.

\section{Surface structure anomaly.}

Since the CBI imaging enables us to observe atom images at their right positions $[4,5]$, we may analyze structure of fine particles directly through the high-resolution CBI images. An advantage of this CBI imaging is quantitative structure analysis of fine particles. The (100) surface of Au particles, for example, is well established to be reconstructed to $n \times 1(n=3,5,7)$ structures differently from the incommensurate reconstruction of the bulk (100) surface [4]. In addition, CBI imaging has revealed that the interplaner distance between the surface top layer and the layer beneath it, is compressed by even $20 \%$ from the one found on the bulk surface [9]. The compression is due to an extra surface tension of the particle which has more broken surface bonds than the bulk surface. 


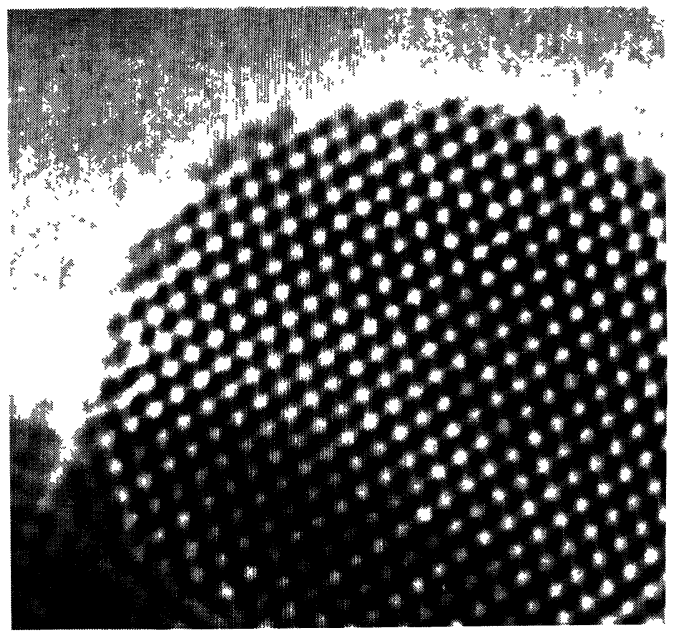

a)

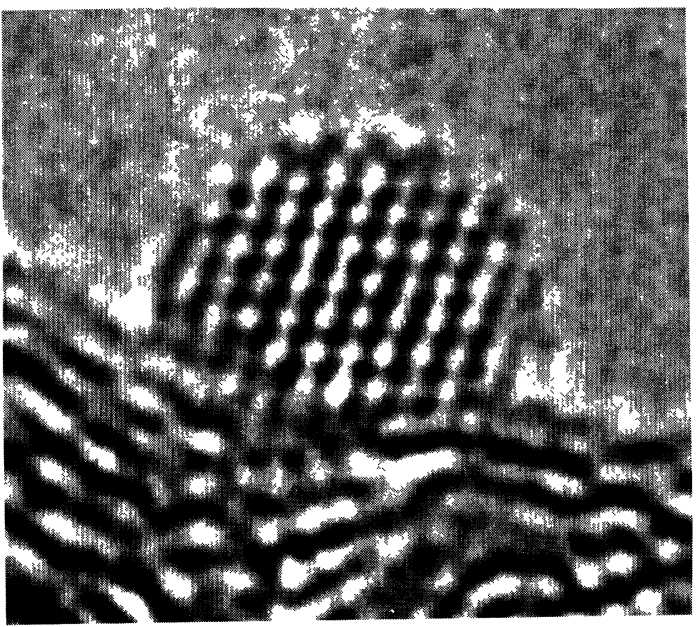

b)

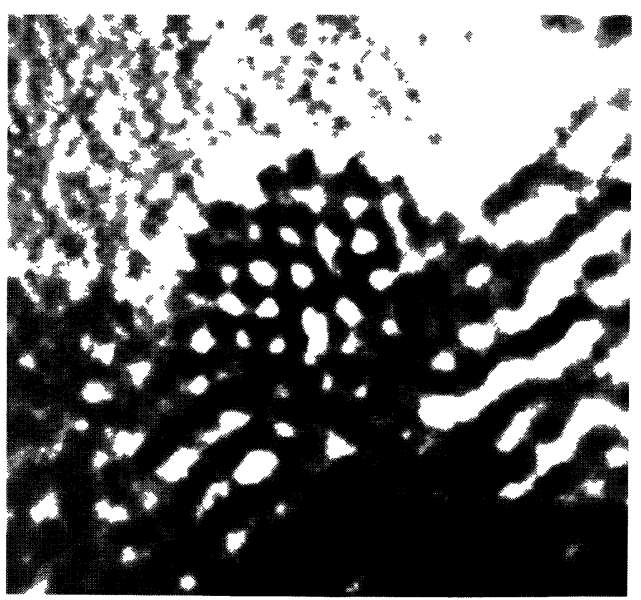

c)

Fig. 2. - Anomalous surface reconstruction on (111) facet and the dimer reconstruction on (100) facet of germanium particle observed by a high-resolution UHV electron microscope. (a) large particle, (b) small particle, and (c) the smallest particle for the surface reconstruction to be stabilized on the (111) facet. 
Another example of the quantitative CBI imaging is structure analysis of an anomalous surface reconstruction observed on (111) facet of fine Ge particles (see Fig. 2) [7]. As the model structure shows (Fig. 3), the reconstruction consists of a stacking-fault. Therefore, the reconstruction is a new type which has been reported to be never stabilized on the bulk (111) surface of Ge.
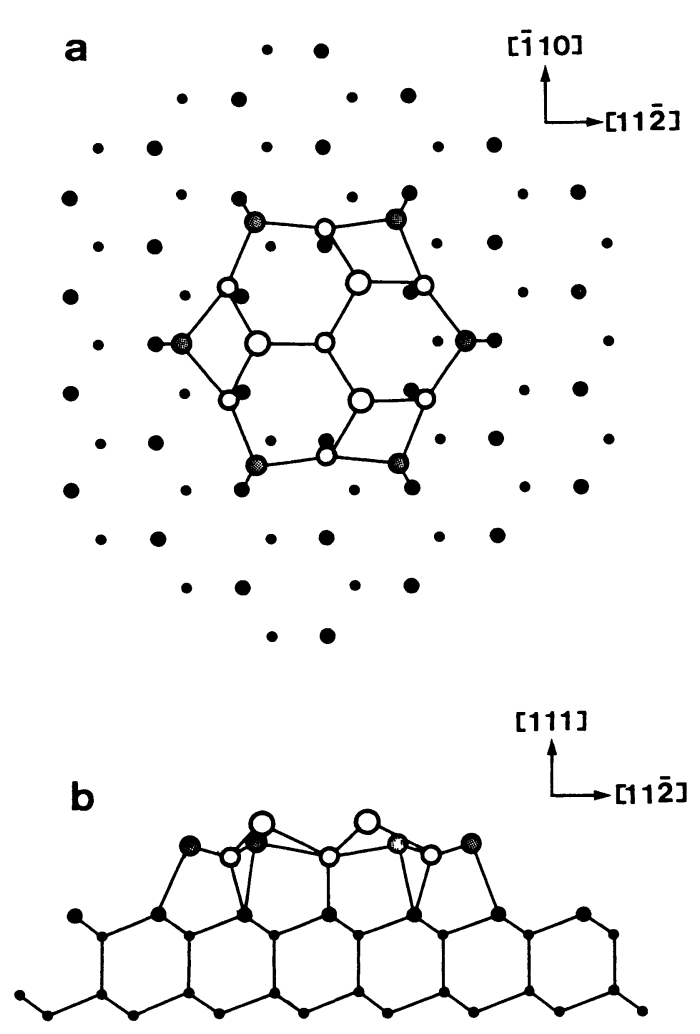

Fig. 3. - A model structure of the anomalous reconstruction on the (111) facet of germanium particle. (a) plan-view, and (b) profile view. Note that three atoms around the center atom locate at the sites of stacking fault.

\section{Dynamic behavior of Sn particle.}

Fine metal particles are often reported to present structural instability during observation. Such instability is often discussed whether it is an intrinsic property of small particles or an artefact induced by strong electron irradiation $[10,11]$. Stability of particles, of course, depends on the interaction with the substrate $[12,13]$, or on the substrate materials, as was discussed by Reiss [14]. There are three kinds of motion of fine particles deposited on a substrate, which might be recognized as instability: 1) structure change (dynamic change in the relative position of atoms), 2) orientation change due to rotational motion (without structure change), and 3) translational displacements on the substrate surface. Mitome et al. has carefully investigated structure instability of Au particles by varying electron beam intensity, substrate temperature, and size of $\mathrm{Au}$ 


\section{L/QC/CS by Cooling}

$2 \mathrm{r}=4 \mathrm{~nm}$

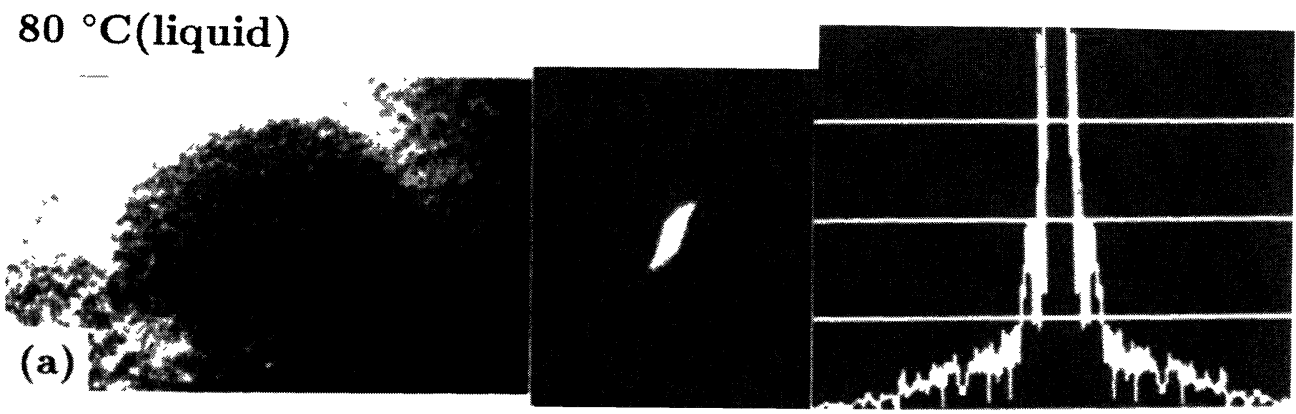

$60-50{ }^{\circ} \mathrm{C}$ (quasi-crys.)

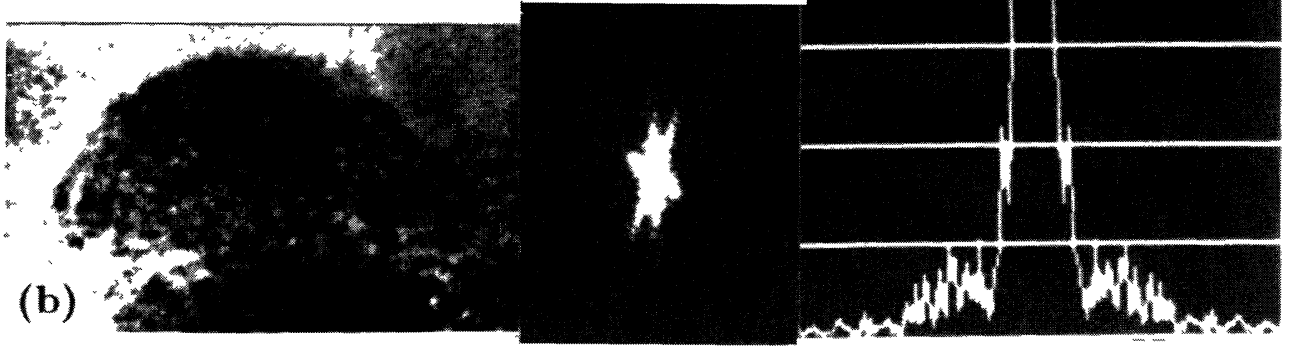

$30{ }^{\circ} \mathbf{C}$ (cryst. solid)

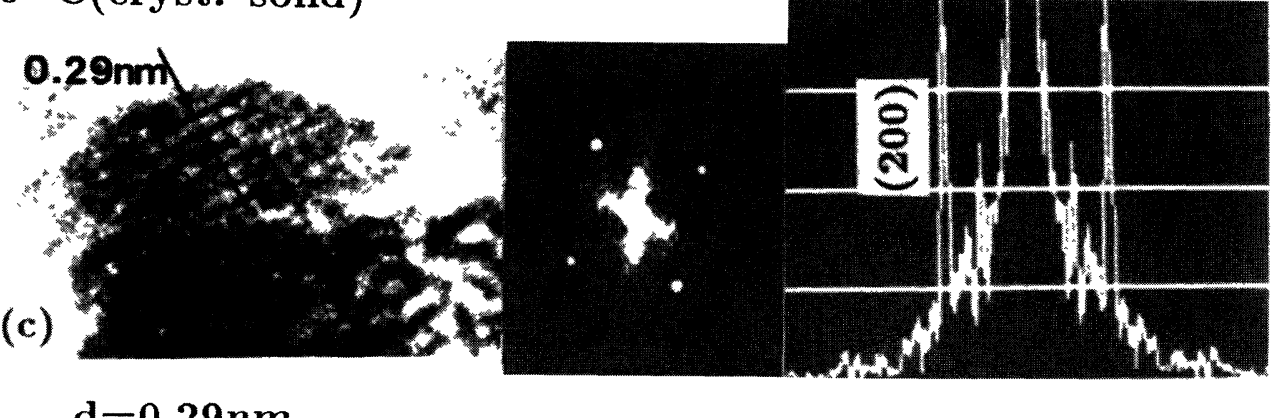

$\mathrm{d}=0.29 \mathrm{~nm}$

Fig. 4. - Phase transition process of a Sn particle. A Sn particle (a) in liquid phase, (b) in an intermediate phase, and (c) in solid phase. Electron microscope image, optical transform of the image (OD), and profile of the OD pattern along a line across the 000 spot. Note that regular lattice fringes in the solid particle, the uniform contrast in the liquid phase, and an irregular image contrast of the particle in the intermediate phase. 
particles to conclude that structural instability, that is, structure change from one stable to the other metastable structures ( $3 \mathrm{~N}-6$ freedom of a particle composed of $\mathrm{N}$ - atoms) is an intrinsic property [15]. Au particles have been observed to translate on the carbon surface, and to move rapidly without changing their structure, since the interaction is week enough. Electron been has also discussed to affect instability through collisions, charging, or thermal excitation. We have observed that intense electron beam irradiation accelerates the instability, but increases particle temperature only by about $30^{\circ} \mathrm{C}$ for $\mathrm{Pb}$ particles deposited on a holey carbon film. The temperature increase is much less than that reported previously [10].

Based on these analysis, structure instability of fine Sn particles has been also investigated insitu, where Sn particles were deposited on lateral surfaces of a holey carbon film in the UHV highresolution electron microscope [8], as well as the experiments of the Au particles described above. Solid Sn particles did not show structure change (see Fig. 4; we do not concern with any change in their orientation) differently from Au particles, because $\mathrm{Sn}$ particles has the body centered tetragonal structure only. As the size of Sn particles decreases, melting temperature decreases. A specific behavior is noticed for particles less than a critical size $(2 r=5 \mathrm{~nm})$ : Solid-liquid phase transition process (Fig. 5) behaves differently from larger particles than the critical size; the transition is sharp for $2 r>5 \mathrm{~nm}$, while an intermediate structure appear between solid and liquid phase for $2 r<5 \mathrm{~nm}$. The electron microscope images of the intermediate structure, which are recorded on a video tape in-situ, suggest that crystalline embrios are generated and annihilated within the particle.

\section{Dynamic behavior of a particle}
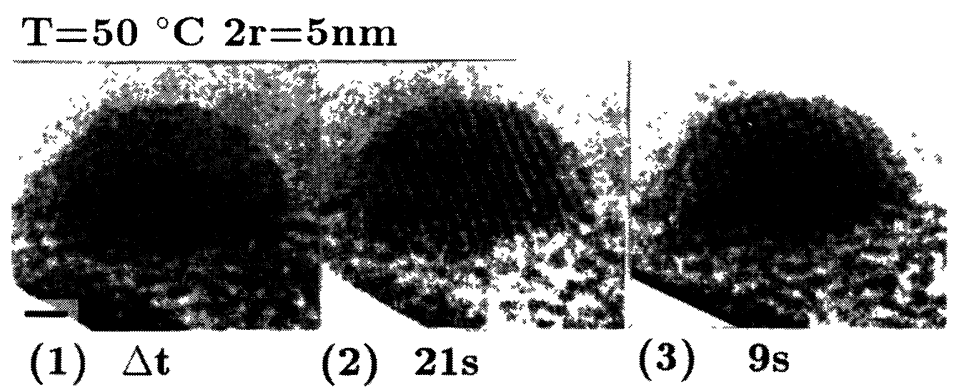

(2) $21 \mathrm{~s}$

(3) $9 \mathrm{~s}$

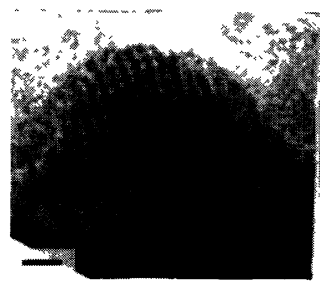

(4) $\approx 1 \mathrm{~s}$

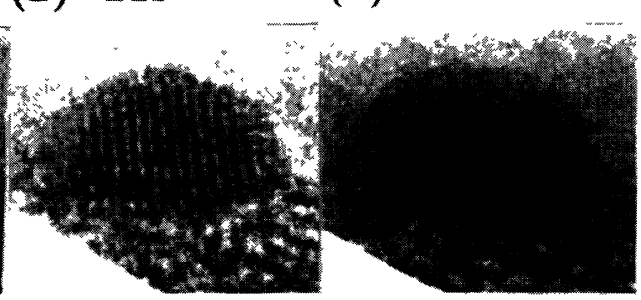

(5) $6 \mathrm{~s}$

(6) $21 \mathrm{~s}$

Fig. 5. - Dynamic behavior of a solid Sn particle at around the critical radius $(2 r=5 \mathrm{~nm})$. Note nearly spherical shape of a Sn particle. Sn particle at the critical size (not shown here) shows irregular dots image at the surface, indicating some disordering of the surface layer. 


\section{Summary and discussion.}

The CBI imaging can give atom images at their right positions independent of thickness. Therefore, credible structure analysis can be done for fine particles having certain irregularities or anomaly. Fine particles have found to have some surface structure anomaly (reconstruction) for $\mathrm{Ge}$ and $\mathrm{Au}$ particles. Mechanism of structure instability of fine particles has not been explained completely, but a new finding of an intermediate phase for Sn particles suggests that structure fluctuate highly at such a small dimension, and structure and phase of such a finite system have to be investigated further in detail with much higher time resolution. Finally it may worth mentioning that the CBI imaging gives a Gaussian type profile image for each atom (instead of an oscillatory profile image by PBI imaging), the CBI method should be powerful for detecting light elements buried in a matrix of heavy elements, e.g. Oxygen atoms in high-Tc material. High-resolution CBI electron microscopy, thus, is expected to reveal new informations of interesting materials.

\section{References}

[1] KuBo R., J. Phys. Soc. Jpn. 17 (1962) 975.

[2] see Microclusters, Eds. S. Sugano, Y. Nihsina and S. Ohnishi (Springer-Verlag, Berlin, 1986).

[3] Cowley J.M., in Diffraction Physics, North-Holland, Amsterdam, 2nd ed. (1981) p. 292.

[4] Mitome M., TANISHIRO Y. and TAKAYANAG K., Ultramicroscopy 33 (1990) 255.

[5] ITOH M., Master Thesis at Tokyo Institute of Technology (1993).

[6] Takayanagi K., Progress Theoretical Physics Suppl. 106 (1991) 249.

[7] MitOME M., TANIÁSHIRO Y. and TAKAYANAGI K., Ultramicroscopy 39 (1991) 382.

[8] OHSHIMA Y. and TAKAYANAGI K., Z. für Physik, in press.

[9] Mitome M., TAKAYANAGI K. and TANiSHIRo Y., Phys. Rev. B42 (1991-I) 7239.

[10] IIJIMA S. and ICHIHASH T., Phys. Rev. Lett. 56 (1986) 616.

[11] BOVIN J.O. and MALM J.O., Z. für Physik D 19 (1991) 293.

[12] AJAYAN P.M. and MARKS L.D., Phys. Rev. Lett. 63 (1989) 279.

[13] GioRgio S. et al., Ultramicroscopy 38 (1991) 1.

[14] ReIss H., J. Appl. Phys. 39 (1968) 5045.

[15] Mitome M., TANishiro Y. and TAKAYANAGi K., Z. für Physik D 12 (1989) 45. 